Supporting Information for

\title{
Large Field-of-View Super-Resolution Optical Microscopy Based on Planar Polymer Waveguides
}

\author{
Anders Kokkvoll Engdahl, Stefan Belle, Tung-Cheng Wang, Ralf Hellmann, \\ Thomas Huser, and Mark Schüttpelz ${ }^{\star}$ \\ ${ }^{\star}$ Corresponding author. E-mail: schuettp@physik.uni-bielefeld.de
}

\section{Details of chip fabrication}

The waveguides are fabricated on a $170 \mu \mathrm{m}$ thick borosilicate microscope coverslip (Menzel Gläser, Germany). In order to create a predetermined breaking point for cleaving at the end of the fabrication process, one side of the coverslip is laser ablated perpendicular to the waveguides. This ablation process is, however, stopped roughly in the middle of the coverslip to ensure that the coverslip still provides sufficient mechanical stability for the subsequent chemical and biological processing steps. The laserprocessing of the waveguides is performed with an ultrashort pulse laser at $1030 \mathrm{~nm}$ wavelength, a pulse duration of $230 \mathrm{fs}$ and a repetition rate of $100 \mathrm{kHz}$ (Light Conversion, Lithuania). The average power of the laser was $4.1 \mathrm{~W}$.

To further ensure a clean surface, a multistage cleaning process is carried out. Each cleaning step has a duration of $8 \mathrm{~min}$, starting with cleaning the coverslip in a 35\% solution of Deconex OP 197 (Borer Chemie, Switzerland) in an ultrasonic bath at a temperature of $60^{\circ} \mathrm{C}$, followed by rinsing twice in deionized (DI) water at room temperature. After rinsing, a second cleaning step with a $100 \%$ solution of Deconex OP 192 (Borer Chemie, Switzerland) in an ultrasonic bath at a temperature of $60^{\circ} \mathrm{C}$ is performed, again followed by rinsing twice in DI water. Subsequent to cleaning the photoresist EpoCore2 (MicroResist, Germany) is spin coated onto the substrate. To finally achieve thin waveguides with a sufficiently small surface roughness and a minimum of defects, the spin coater was operated at a speed of $5000 \mathrm{rpm}$. The solvent content in the photoresist is reduced by soft-baking the sample on a hot plate at $50{ }^{\circ} \mathrm{C}$ for $2 \mathrm{~min}$ with a subsequent heating step using a temperature ramp of $8 \mathrm{~K} \mathrm{~min}^{-1}$ up to $90^{\circ} \mathrm{C}$, followed by baking at $90^{\circ} \mathrm{C}$ for $2 \mathrm{~min}$. Then, the photoresist is exposed to UV light using a mask aligner (EVG Group, Austria) at a dose of $103 \mathrm{~mJ} \mathrm{~cm}^{-2}$ (broadband illumination, no bandpass filter used). Immediately after exposure to UV light, the resist film is baked at $85^{\circ} \mathrm{C}$ for $3 \mathrm{~min}$ and after cooling to room temperature the sample is developed for $50 \mathrm{~s}$ in a ready-to-use mr-Dev 600 developer (MicroResist, Germany) and thoroughly rinsed with isopropyl alcohol. For mechanical and chemical stabilization of the developed waveguides an additional hard-baking step at $130{ }^{\circ} \mathrm{C}$ for $30 \mathrm{~min}$ is performed. As a last processing step, the waveguide chip is cleaved at the laser-ablated line. The cleaving process provides an end facet with a low roughness and therefore with superior optical coupling efficiency. The waveguides manufactured according to the parameters described above had a mean height of $1198.2 \mathrm{~nm}$, a standard deviation of $4.4 \mathrm{~nm}$, and the surface roughness is $\mathrm{Sa}=(5.5 \pm 3.7) \mathrm{nm}$. 


\section{Inverted waveguide microscope setup}

A sketch of the microscope setup that complements the polymer waveguide chips is shown in Fig. S1. The microscope consisted of an inverted detection path used for fluorescence detection, an upright detection path used for inspection of the waveguide facets, and a sideways path for coupling of the laser beam into the waveguides.

An Argon-Krypton ion laser was used as a laser source (Innova 70C Spectrum, Coherent Inc.). Laser lines were selected using an acoustic-optical tunable filter. A rotating diffuser was placed in the middle of a 1:1 Keplarian telescope consisting of two $50 \mathrm{~mm}$ focusing lenses (AC254-050-A-ML, Thorlabs Inc). The rotating diffuser was custom built using an aceton-etched polypropylene Petri dish bottom cut to a circle and attached to a $12 \mathrm{~V}$ computer fan. After the rotating diffuser the beam was directed to the coupling optics on top of a Thorlabs NanoMax 300 xyz-piezo stage (MAX312D, Thorlabs Inc). A long working distance $20 \times, 0.25$ NA objective lens (SLMPlan $20 \times 0.25$ Olympus) was used to focus the beam onto the end facet of a waveguide. The piezo stage with the controller (MDT693B, Thorlabs) was used to position the coupling beam. The controller box could be externally controlled by means of USB from a computer to change voltages over the piezo elements, in order to alternate the focus spot when not using the diffuser, and to optimize coupling efficiency. The different intensity profiles occuring in a waveguide are shown in Fig. S3. Alternatively, a folding mirror could be swapped down to direct the laser beam to an epi-illumination pathway, using a dichroic mirror in the detection pathway. The sample stage consisted of a custom-built chip holder resting on top of a y-axis displacement stage for selecting different waveguides. In order to position the sample relative to detection optics without displacing the coupling beam from the current waveguide, the sample stage with y-axis displacement stage and the coupling optics were mounted on top of two ( $\mathrm{x}$ and $\mathrm{y}$ ) mechanical actuators (Spindler \& Hoyer microcontroller). The optical path of the detection optics was located $\sim 1 \mathrm{~cm}$ away (x direction) from the upright pathway. The inverted approach consisted of an objective lens (UCPlanFL N $20 \times 0.7$ NA, Olympus, air for $20 \times$ measurements, or an UPlanApo $60 \times 1.20$ NA water, Olympus, for $60 \times$ measurements), a dichroic mirror (FF410/504/582/669-Di01-25x36, Semrock, to enable epi-illumination). The emission filters consisted of RazorEdge 647 long pass (Semrock), and HQ700/75 M band pass (Chroma) for $647 \mathrm{~nm}$ excitation, and a Razor Edge 568 long pass (Semrock) with a Brightline 593/40 band pass (Semrock) for $568 \mathrm{~nm}$ excitation. A $180 \mathrm{~mm}$ tube lens was used in order to image the sample onto a PCO Edge 4.2 sCMOS camera. The upright pathways was used for collection of scattered light and end facet inspection. It consisted of an objective lens (PLAN 10×0.25 NA (Olympus) for brightfield inspection), 90/10 beam splitter (Thorlabs BSN10R) for LED brightfield band pass or OD3 filters, a $160 \mathrm{~mm}$ tube lens (Linos AC f-160), and an IDS $\mu$ Eye 3060CP CMOS camera. These components were attached to holders for the microrod system, which was built on an Olympus BXFM-ILHS mount frame with z-stage, and fastened to the optical table. 
(a)

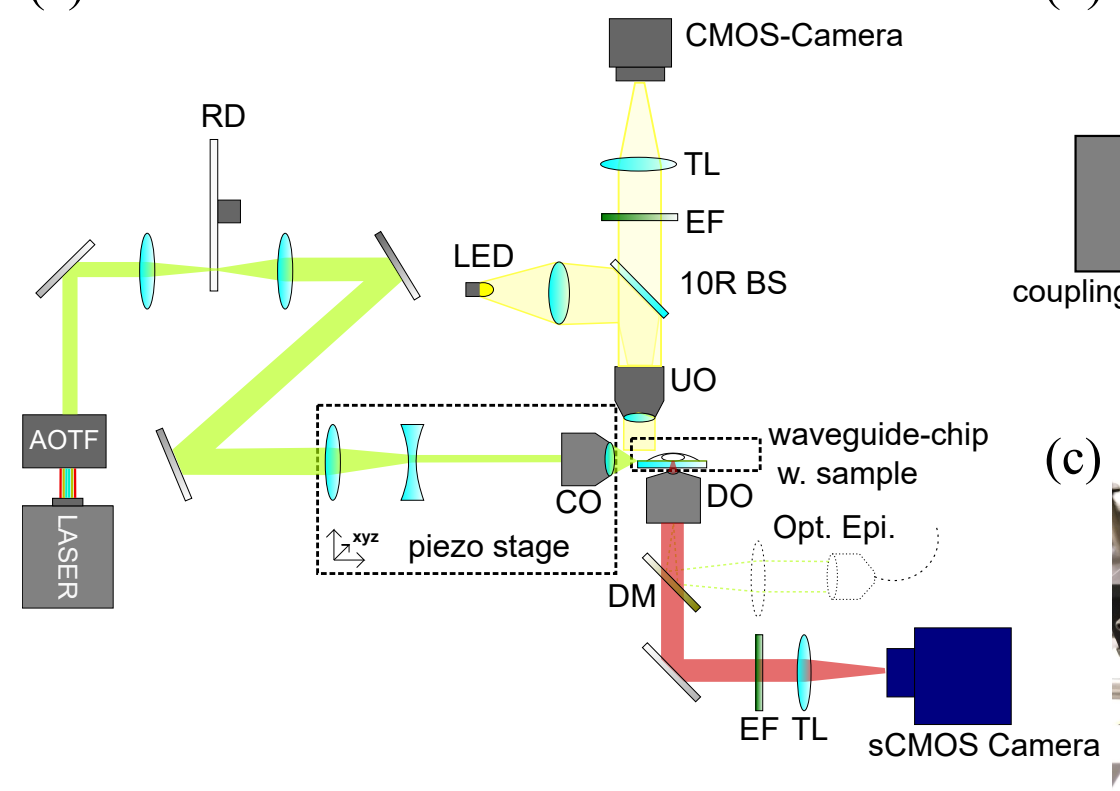

(b)

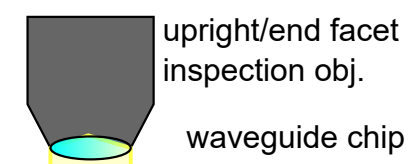
with sample

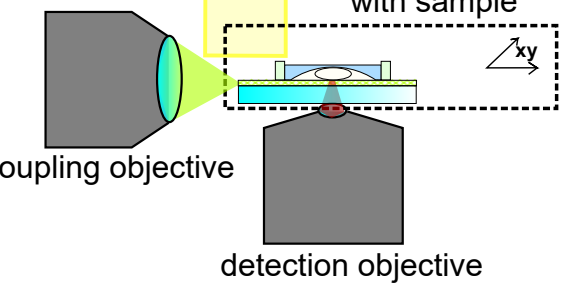

(c)

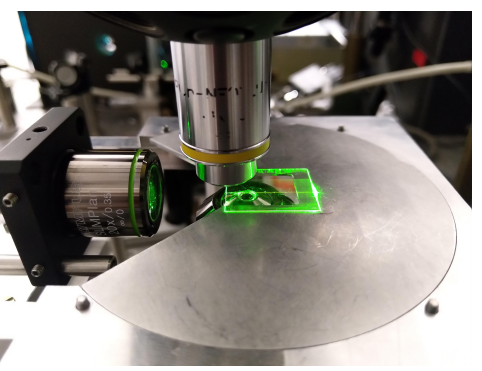

Figure S1: The waveguide fluorescence microscope. A schematic of the setup with sideways end facet coupling is shown in (a). It includes an upright pathway that can be used for visual inspection of the waveguide facets or alternatively for fluorescence detection. An enlarged view of the sample area is shown in (b) and a photo of the sample stage with laser light coupled into a waveguide is shown in (c).

\section{Simulations}

\subsection{Penetration depth of the evanescent field}

We used Meep, 1 an open-source software using the finite-difference-time-domain method in order to simulate light propagating through a waveguide with the geometry matching our chip. The aim was to estimate the decay of the evanescent field and its characteristic field depth for the wavelengths used in our experiments. Meep allowed us to create a cell with a 2D geometry of the waveguide, since the waveguide widths are so much larger than the height, that its effects on the propagating wave can be neglected. Geometries were created as seen in in Fig. S2(a),(b) with a glass substrate, and water sample chamber. The propagating wave was created as a continuous source with origin at the center of the waveguide at the far left end of the simulation cell. At the far right end of the waveguide, we probed the resulting electric field. The electrical field was probed so to find the point at the waveguide-sample chamber boundary at the last $10 \mu \mathrm{m}$ of the waveguide with the largest amplitude. The decay of this amplitude from the boundary to $0.5 \mu \mathrm{m}$ above the waveguide at this point was then fitted against an exponential decay (a linear regression of the natural logarithm of the amplitudes). The resulting fit is shown in Fig. S2 (d). At the point of the penetration depth, we compared the amplitude with the highest amplitude inside the waveguide, to also study how much of the electric field is actually carried into the sample. A comparison of these findings for the two different waveguide heights $(0.85 \mu \mathrm{m}$ and $1.66 \mu \mathrm{m})$ and wavelengths are shown in Fig. S2(e),(f). The penetration depths found from these simulations are displayed in Table $\mathbf{S 1}$. 

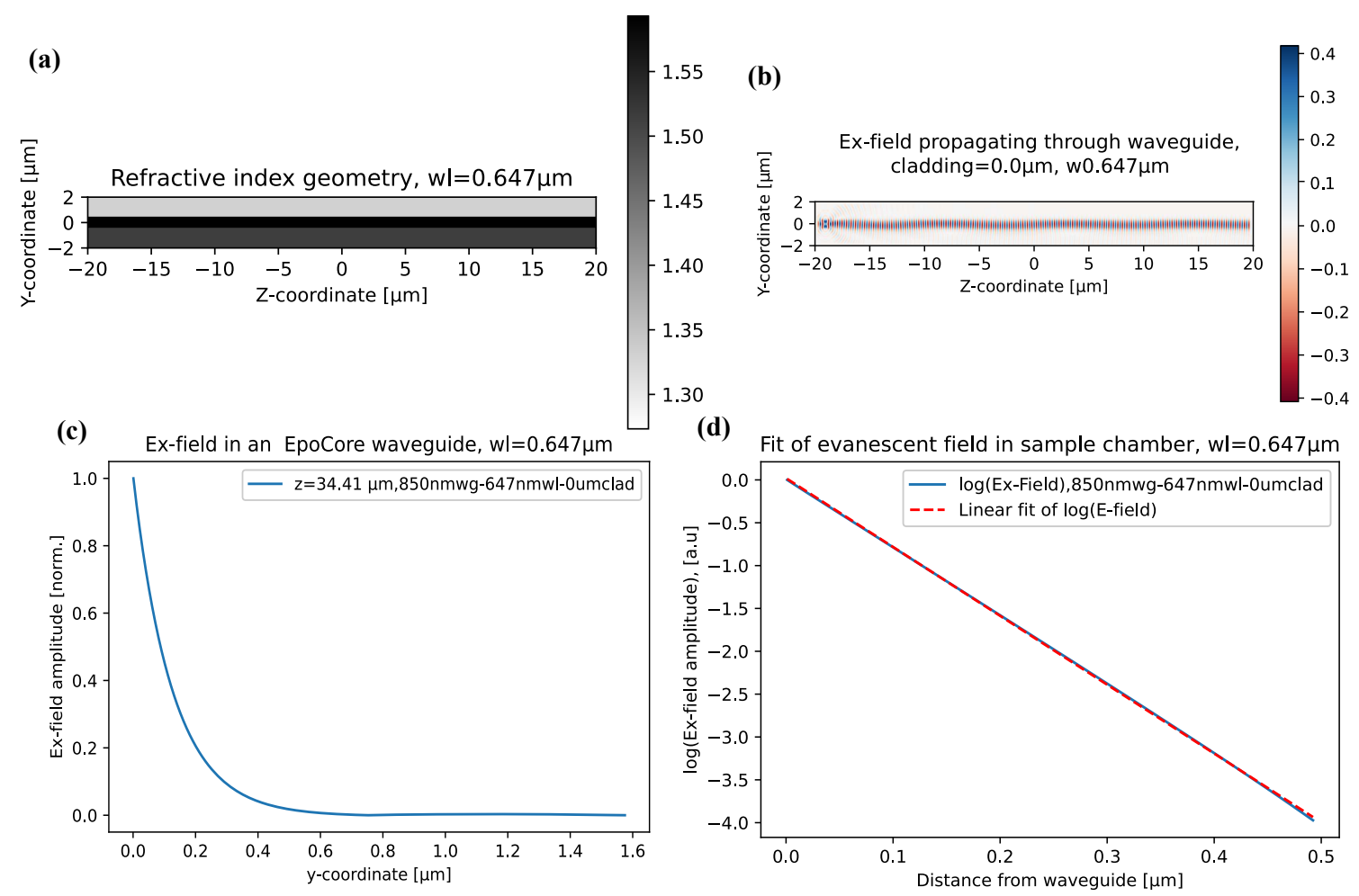

(d)

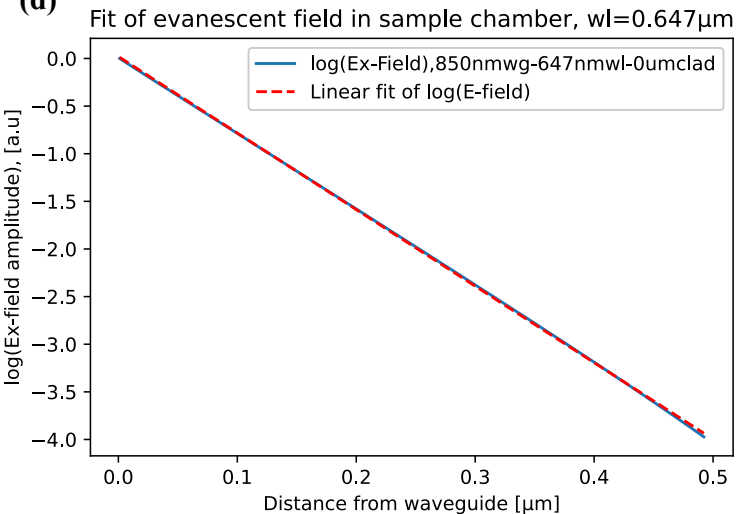

(e)
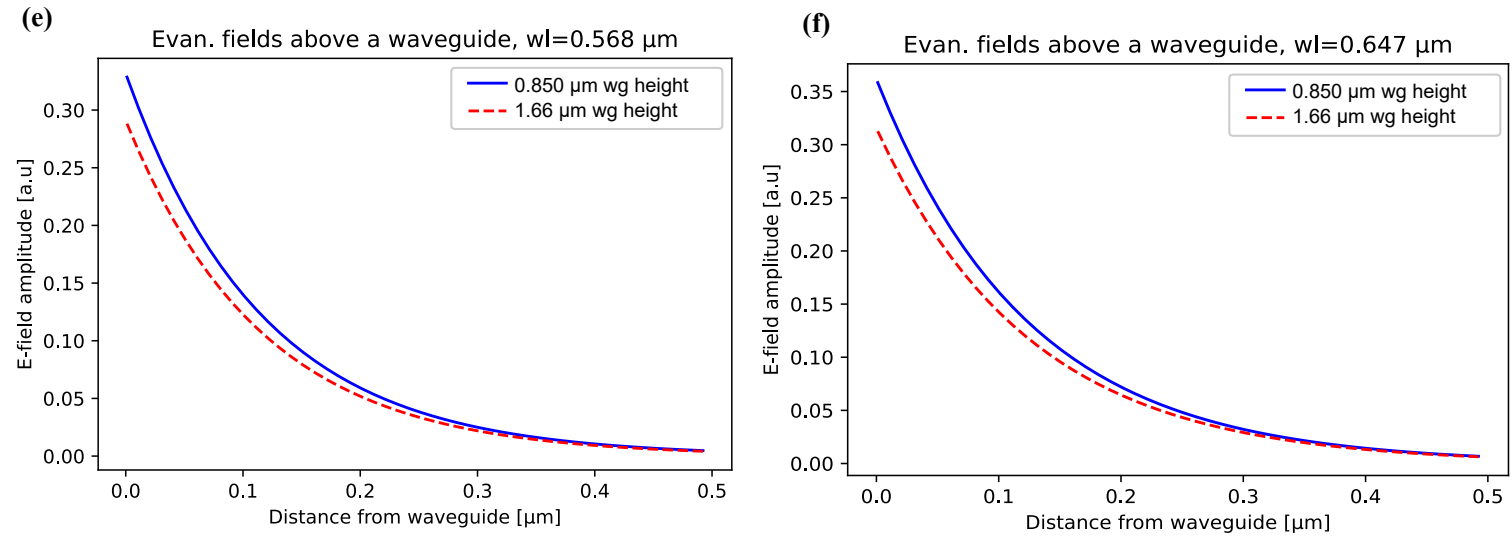

Figure S2: Results of simulations of light propagating through the waveguide. (a) The geometry of the 2D cell used for simulations. The refractive indices are shown in grayscale. (b) A map of the electric field in the waveguide, once the simulation is stopped. (c) A vertical slice close to the right end of the cell shows the decay of the evanescent field in the sample chamber, as a function of distance from the waveguide. This data is fitted using linear regression of its natural logarithm, shown in (d). (e) and (f) show decays of evanescent fields from EpoCore1 and EpoCore2 waveguides used in this study for $568 \mathrm{~nm}$ and $647 \mathrm{~nm}$, respectively. 
Table S1: Penetration depths for the different wavelengths for the waveguide heights used in this study. $d$ is penetration depth [nm], $A$, [a.u], is the corresponding fraction of the electric field that is carried to the evanescent tail.

\begin{tabular}{c|cccc} 
& \multicolumn{2}{|c}{$568 \mathrm{~nm}$} & \multicolumn{2}{c}{$647 \mathrm{~nm}$} \\
Waveguide height & $d[\mathrm{~nm}]$ & $A$ & $d[\mathrm{~nm}]$ & $A$ \\
\hline $0.85 \mu \mathrm{m}$ & 116 & 0.122 & 124 & 0.133 \\
$1.20 \mu \mathrm{m}$ & 107 & 0.09 & 127 & 0.10 \\
$1.66 \mu \mathrm{m}$ & 116 & 0.107 & 126 & 0.116
\end{tabular}

\subsection{Estimation of coupling efficiency}

We estimated the coupling loss for $647 \mathrm{~nm}$ light by calculating the Fresnel coefficients for transmission into a material with the refractive index of EpoCore (1.5928) for entrance angles from 0 degrees up to the numerical aperture of the coupling lens (0.25). Each angular value was weighted with a fraction of the power of a Gaussian beam profile with a full width at half maximum (FWHM) corresponding to the three scenarios (measured before the coupling objective using a beam profiler, and scaled with a formula

$$
\mathrm{FWHM}_{\text {afterobj }}=\frac{r_{\text {exit }} \times \mathrm{FWHM}_{\text {beforeobj }}}{r_{\text {entrance }}} .
$$

Here $r_{\text {exit }}$ is the radius of the exit pupil of the objective lens and $r_{\text {entrance }}$ is its entrance pupil radius. This fraction of transmission resulting from these calculations was multiplied with a second fraction, the fraction of the beam that hits the end facet. This value was calculated by first measuring the FWHM of the beam focus after the coupling objective by obtaining the diffracted spot at an end facet with the upright part of the microscope (measured with a $50 \times, 0.5 \mathrm{NA}$ air objective). The fraction hitting the end facet of the waveguide was then calculated as

$$
\int_{0}^{h / 2} \frac{1}{\sigma \sqrt{2 \pi}} \exp \left(-\frac{x^{2}}{2 \sigma^{2}}\right) \mathrm{d} x,
$$

an integral over a normalized Gaussian profile with its corresponding FWHM, from 0 (center of beam) to half of the height of the waveguide $h$, and doubled. The fraction of the beam that hits the waveguide was multiplied with the fraction calculated to be transmitted into the waveguide. The results are shown in the Table $\mathbf{S 2}$.

\section{Preparation of polymer waveguide chips}

Fresh EpoCore waveguide chips were cleaned the following way: using $70 \%$ ethanol in water in a sonicator bath at room temperature for $5 \mathrm{~min}$ or using a $0.1 \%$ sodium dodecyl sulfate (SDS) in water solution in a sonicator bath. After cleaning, chips were rinsed with water or ethanol and blow dried using pressurized nitrogen. 

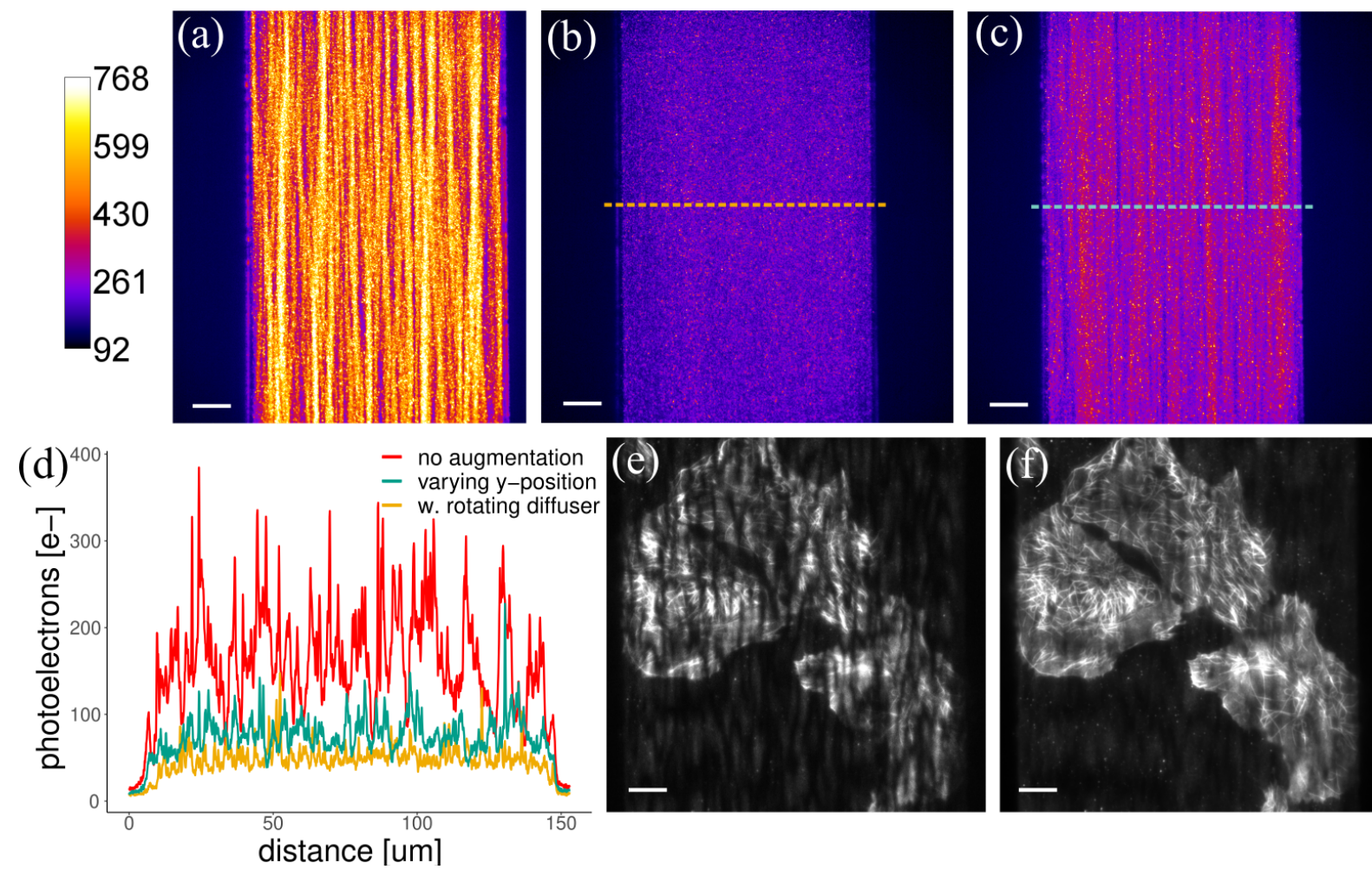

Figure S3: TIRF intensity profiles from an $140 \mu \mathrm{m}$ wide and $0.9 \mu \mathrm{m}$ high waveguide, displaying the different coupling modalities. (a) A display of the streak pattern of the waveguide, when simply coupling with a fixed, focused beam. Calibration bar shows gray values. (b) Using a rotating diffuser to remove the coherence of the light entering the waveguide. (c) Varying the focus spot along the end facet of the waveguide evens out the streak patterns over time. (d) Line profiles of (a)-(c). (e) U2OS cells on a $90 \mu \mathrm{m}$ wide waveguide stained for tubulin, imaged without any beam augmentation, and (f), by varying the focus spot as done in (c). Exposure time was $1 \mathrm{~s}$ in all cases, and the autofluorescence of a fresh waveguide was used for the measurements in (a)-(c). The waveguide was covered with PBS and was measured with a $60 \times, 1.2 \mathrm{NA}$ water objective lens. Scale bars are $20 \mu \mathrm{m}$ in (a)-(c) and $10 \mu \mathrm{m}$ in (e), (f). The color bars are identical in (a)-(c). 
Table S2: Highest possible coupling efficiencies into the waveguides for $647 \mathrm{~nm}$ light using a $20 \times, 0.25$ NA objective lens.

\begin{tabular}{c|cc} 
Waveguide height & no diffuser & coarse diffuser \\
\hline $0.858 \mu \mathrm{m}$ & $14.1 \%$ & $6.2 \%$ \\
$1.200 \mu \mathrm{m}$ & $19.0 \%$ & $8.7 \%$ \\
$1.660 \mu \mathrm{m}$ & $24.7 \%$ & $11.9 \%$
\end{tabular}

\section{Waveguide characterization experiments}

\subsection{Bleaching experiments}

An unused $0.9 \mu \mathrm{m}$ high and $240 \mu \mathrm{m}$ wide EpoCore1 waveguide was first cleaned using the protocol described above. Following optimization of the coupling efficiency of light into the waveguide, the waveguide was prebleached for $10 \mathrm{~min}$ using $647 \mathrm{~nm}$ at $16 \mathrm{~mW}$ laser power using the piezo controller with the function generator to vary the position of the focused beam. The detection area on the waveguide was $1 \mathrm{~cm}$ away from the end facet using the inverted detection path and a $20 \times$ objective. An image of the waveguide autofluorescence was recorded every $10 \mathrm{~s}$ with $1 \mathrm{~s}$ of camera exposure. To compare intensities, the intensity of the waveguide was recorded before and after bleaching with optimal coupling conditions, and the mean of the gray value signal was converted to photoelectrons, showing that $90 \%$ of the fluorescence signal had been bleached. Following another 50 min of bleaching, a total reduction of $97 \%$ of the waveguide autofluorescence could be reached. The same waveguide was re-recorded 24 hours later using the same protocol, showing that the fluorescent signal had recovered to $11 \%$ the intensity of the initial measurement.

\subsection{Attenuation experiments}

A fresh EpoCore2-chip with $1.0 \mu \mathrm{m}$ thick waveguides was cleaned using the protocol described above, and a transparent PDMS chamber was placed on top of the chip to house a liquid sample. A $260 \mu \mathrm{m}$ wide waveguide was prebleached for $30 \mathrm{~min}$ using $9 \mathrm{~mW} 568 \mathrm{~nm}$ at the back focal plane of the coupling objective, and then for a similar duration using $647 \mathrm{~nm}$ with $6 \mathrm{~mW}$ power, using the rotational diffuser in the beam path to even out mode patterns in the waveguide. The same intensities were used for the subsequent measurements. Afterwards, $0.1 \mu \mathrm{m}$ fluorescent TetraSpeck microspheres (T7279, Invitrogen) were applied on the waveguide chip using a 1:500 dilution in phosphate-buffered saline (PBS), and left to settle for two hours. After washing thrice with ultrapure water and an addition of water, the fluorescent beads were imaged along the waveguide, from a position $0.5 \mathrm{~cm}$ to a position $2 \mathrm{~cm}$ away from the coupling end facet, with $0.3 \mathrm{~mm}$ displacement between each image. For this measurement the inverted detection path using a $20 \times, 0.7$ NA objective lens was used. Images were taken using $200 \mathrm{~ms}$ exposure time. The measurements were repeated three times for each wavelength. The fluorescentce intensities of the beads were analysed with the ThunderSTORM Fiji plugin, $\underline{2}$ normalized for each measurement and plotted together in Fig. S4. An exponential decay function was fitted against this data, 
resulting in an estimated $(0.92 \pm 0.05) \mathrm{dB} \mathrm{cm}^{-1}$ (19\% loss of intensity per $\left.\mathrm{cm}\right)$ for $568 \mathrm{~nm}$ excitation, and a $(0.60 \pm 0.05) \mathrm{dB} \mathrm{cm}^{-1}(13 \%$ loss of intensity per $\mathrm{cm})$ for $647 \mathrm{~nm}$ excitation.
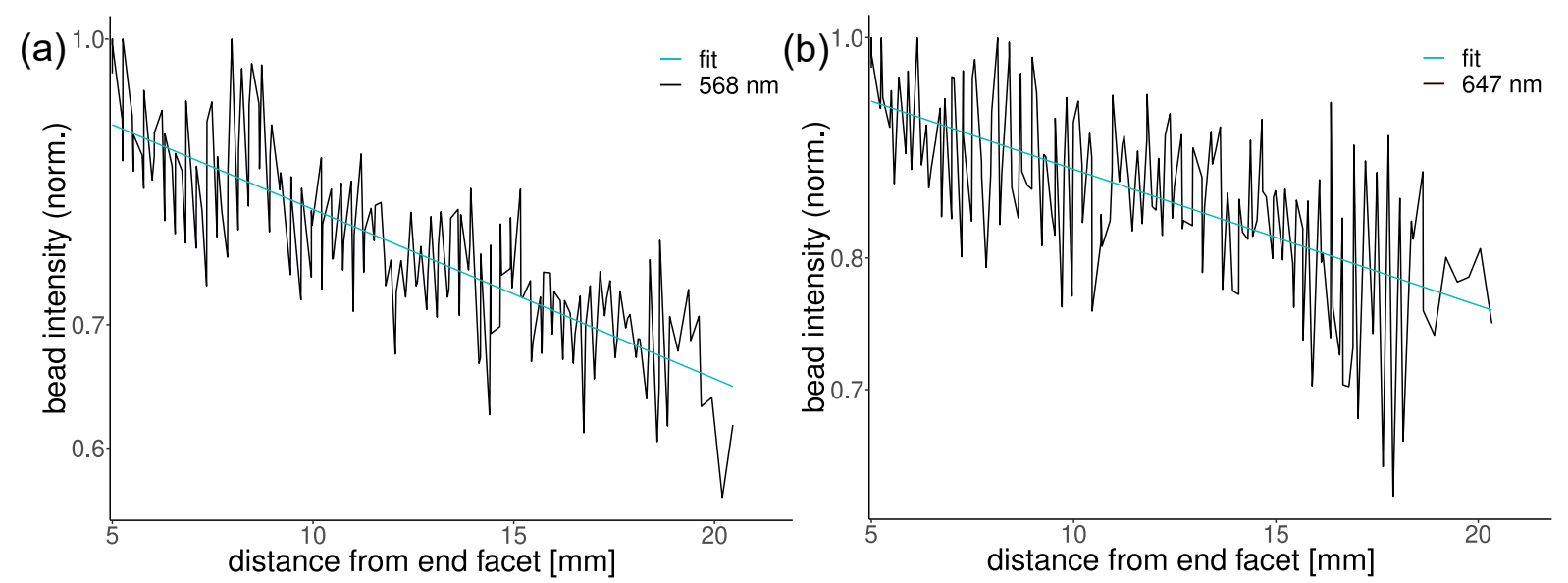

Figure S4: Attenuation of fluorescence intensity of Tetraspeck beads under (a) $568 \mathrm{~nm}$ and (b) $647 \mathrm{~nm}$ excitation on a waveguide, plotted as a function of the distance from the end facet. A $260 \mu \mathrm{m}$ wide, $1.0 \mu \mathrm{m}$ tall EpoCore2 waveguide was used for the experiments. An exponential decay function is fitted to the data.

\section{Cell sample preparation}

EpoCore waveguide chips were cleaned in a sonicator bath using a $0.1 \%$ SDS solution in ultrapure water for $5 \mathrm{~min}$ and rinsed with reagent grade ethanol, then blow dried using pressurized nitrogen. Afterwards they were placed in sterilized Petri dishes, and sample chambers made from PDMS were added on top of the chips. They were grown with U2OS cells $\left(10,000\right.$ cells $\left.\mathrm{cm}^{-2}\right)$ in the PDMS chamber, supplemented with 10\% Fetal bovine serum (FBS) in Dulbecco's Modified Eagle's medium (DMEM), and kept in $5 \% \mathrm{CO}_{2}$ climate incubator overnight. In the next morning, cells were fixed using $0.2 \%$ glutaraldehyde and 4\% paraformaldehyde in PBS solution for $15 \mathrm{~min}$ at room temperature. Afterwards the fixation solution was aspirated and washed three times with PBS solution. Staining was done as follows: 10 min with $0.1 \%$ Triton-X100 in water, aspirated, three times washed, then glutaraldehydeinduced autofluorescence was induced with $0.1 \% \mathrm{NaBH}_{4}$ in PBS for $7 \mathrm{~min}$, then aspirated and washed thrice. Then followed blocking with 5\% Bovine Serum Albumin (BSA) in PBS for 45 min. The staining solution consisted of 1:200 diluted mouse anti-alpha-tubulin IgG (A11126, Invitrogen) and 1:200 diluted mouse anti-beta tubulin IgG (T8328, Sigma-Aldrich) in 1\% BSA with $0.025 \%$ Triton-X100 in PBS solution and was incubated overnight at $4^{\circ} \mathrm{C}$. After aspiration and three times washing with PBS, the secondary solution, goat anti-mouse IgG conjugated with Alexa Fluor 647 (A-21237, Invitrogen) together with 1:20 CF568-Phalloidin (00044, Biotium) for actin staining, also in 1\% BSA and $0.025 \%$ Triton-X100 solution was incubated over $90 \mathrm{~min}$ at room temperature. Lastly, the solution was aspirated, and the samples washed five times. The imaging conditions we used are listed in Table S3. 
Table S3: Imaging conditions for micrographs used in this paper.

\begin{tabular}{|c|c|c|c|c|c|c|c|}
\hline Figure & Sample, Target, Medium & Fluorophore, Conjugate & \# Images & $\lambda[\mathrm{nm}]$ & Power $[\mathrm{mW}]$ & Objective Lens & Exp. Time [ms] \\
\hline Fig. 2 & U2OS, actin, $1 \times$ PBS & CF568, phallodin & 1 & 568 & 12 & $60 \times$ w, NA 1.20 & 100 \\
\hline Fig. 3 & Clean waveguide, $1 \times$ PBS & n.a. & 1 & 647 & 16 & $20 \times$, NA 0.7 & 1000 \\
\hline Fig. 4 (green) & U2Os, actin, $1 \times$ PBS & CF568, phalloidin & 1 (wf), 200 (SRRF) & 568 & 12 & $20 \times$, NA 0.7 & 100 \\
\hline Fig. 4 (magenta) & U2OS, actin, $1 \times$ PBS & $\begin{array}{l}\text { Alexa Fluor } 647, \\
\text { goat anti-mouse IgG }\end{array}$ & 1 (wf), 200 (SRRF) & 647 & 11 & $20 \times$, NA 0.7 & 100 \\
\hline Fig. 5 (green) & $\mathrm{U} 2 \mathrm{OS}$, actin, $1 \times$ PBS & CF568, phalloidin & 1 (wf), 200 (SRRF) & 568 & 9 & $60 \times$ w, NA 1.20 & 100 \\
\hline Fig. 5 (magenta) & U2OS, actin, $1 \times$ PBS & $\begin{array}{l}\text { Alexa Fluor 647, } \\
\text { goat anti-mouse IgG }\end{array}$ & 1 (wf), 200 (SRRF), & 647 & 11 & $60 \times$ w, NA 1.20 & 100 \\
\hline
\end{tabular}

\section{Software}

$\mu$ Manager (version 1.4.22), ${ }^{3}$ was used for image registration using the PCO 4.2 camera. Post processing was done using ImageJ with the Fiji package. For a SRRF ${ }^{4}$ image reconstruction, wide-field images were acquired using $100 \mathrm{~mm}$ exposure times. 200 frames were used for each reconstruction using ring radius 0.5 , radiality magnification 5, 6 axes in ring. For Fourier ring correlation, image stacks were split into odd and even frames, from which SRRF reconstructions were made. NanoJ-SQUIRREL ${ }^{5}$ was used on the insets shown in Fig. 4(b), dividing the images into grids of $10 \times 10$ squares, with the Fourier Ring Correlation being calculated for each square. This procedure was repeated for the inset shown in Fig. 5(b).

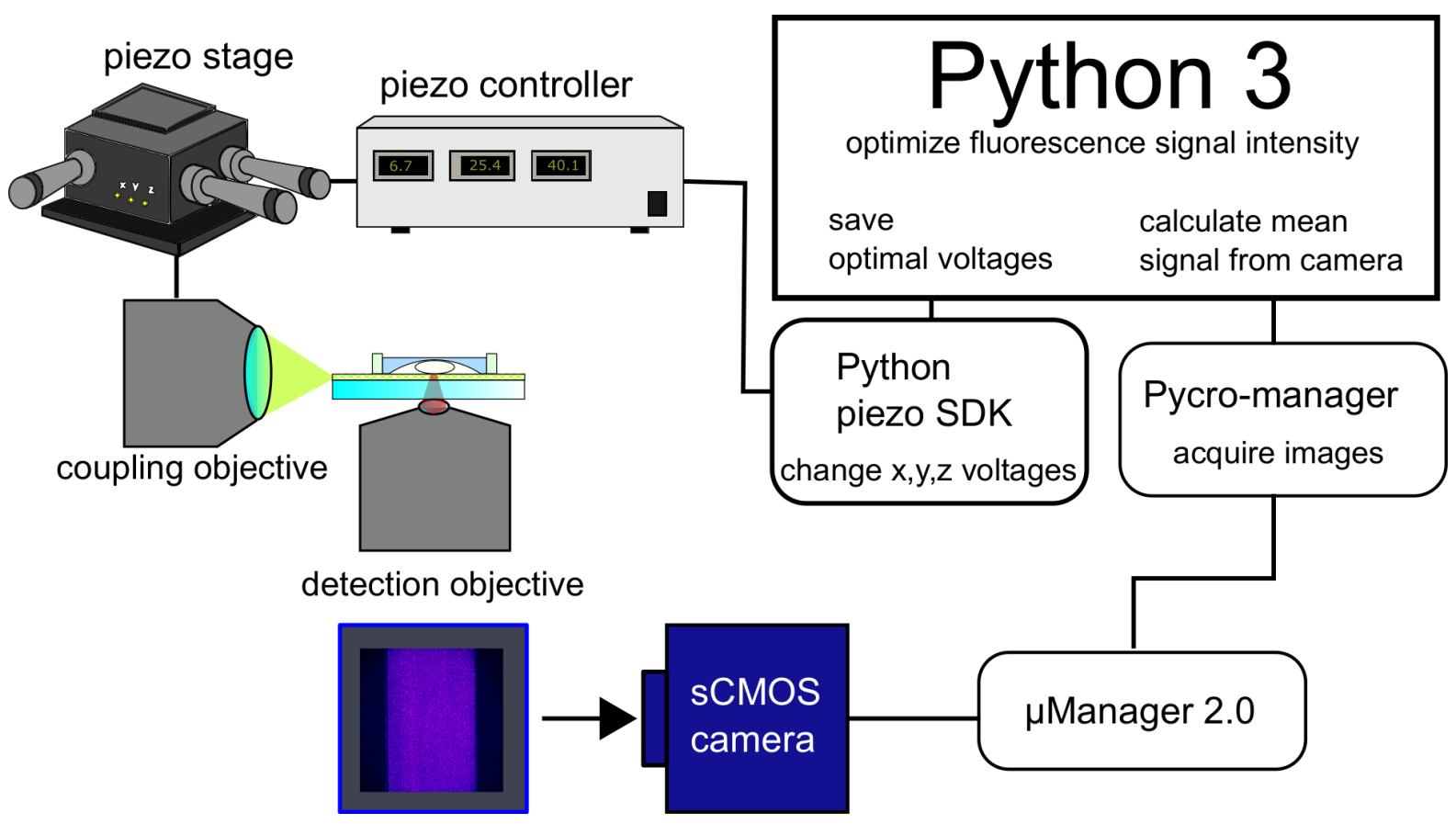

Figure S5: Scheme for the setup that was used to automate and optimize coupling.

\subsection{Automatic coupling script}

We created a system that automatically can couple into a desired waveguide, and optimize the fluorescent signal on the camera detector. This is done by connecting the detector signal from the PCO camera that is captured using the $\mu$ Manager software application with the Thorlabs NanoMax300 piezo stage that 
can move the coupling objective in three dimensions. An overview of the system with its components is shown in Fig. S5. The automatic coupling script is written in Python 3, since the Piezo controller (Thorlabs MDT693B, USA) has a Python 3 SDK. The other central component that we use, is PycroManager. ${ }^{[6}$ It allows us to manipulate $\mu$ Manager to acquire images that can be accessed in Python. When the script is executed, the piezo stage will proceed to move the beam focus across range of voltages ( $75 \mathrm{~V}$ relating to a total range of approximately $22 \mu \mathrm{m}$ ); at each spot the PCO camera acquires an image of the fluorescent structure, and the script calculates the mean intensity of each image. By doing this, a range of image intensities at the different focus spots can be calculated, and the spot with the highest mean intensity is selected as the optimum. This is repeated twice with different voltage step sizes, each time around a new middle. This can be done for all three voltage channels, $x, y$, and $z$, but the $\mathrm{z}$ - and the $\mathrm{y}$ channels are usually the most important, since a waveguide only has a height of 0.8-2 $\mu \mathrm{m}$ (corresponding to the $\mathrm{z}$ channel), but the waveguide width can vary between $10-600 \mu \mathrm{m}$.

\section{References}

[1] Oskooi, A. F.; Roundy, D.; Ibanescu, M.; Bermel, P.; Joannopoulos, J.; Johnson, S. G. Meep: A flexible free-software package for electromagnetic simulations by the FDTD method. Comput. Phys. Commun. 2010, 181, 687 - 702, DOI: $10.1016 /$ j.cpc. 2009.11 .008$.

[2] Ovesný, M.; Křížek, P.; Borkovec, J.; Švindrych, Z.; Hagen, G. M. ThunderSTORM: a comprehensive ImageJ plug-in for PALM and STORM data analysis and super-resolution imaging. Bioinformatics 2014, 30, 2389-2390, DOI: 10.1093/bioinformatics/btu202.

[3] Edelstein, A.; Tsuchida, M.; Amodaj, N.; Pinkard, H.; Vale, R.; Stuurman, N. Advanced methods of microscope control using $\mu$ Manager. J. Biol. Methods 2014, l, DOI: 10.14440 / jbm. 2014.36.

[4] Gustafsson, N.; Culley, S.; Ashdown, G.; Owen, D. M.; Pereira, P. M.; Henriques, R. Fast live-cell conventional fluorophore nanoscopy with ImageJ through super-resolution radial fluctuations. Nat. Commun. 2016, 7, 12471, DOI: 10.1038 /ncomms 12471.

[5] Laine, R. F.; Tosheva, K. L.; Gustafsson, N.; Gray, R. D. M.; Almada, P.; Albrecht, D.; Risa, G. T.; Hurtig, F.; Lindås, A.-C.; Baum, B.; Mercer, J.; Leterrier, C.; Pereira, P. M.; Culley, S.; Henriques, R. NanoJ: a high-performance open-source super-resolution microscopy toolbox. J. Phys. D: Appl. Phys. 2019, 52, 163001, DOI: 10.1088/1361-6463/ab0261.

[6] Pinkard, H.; Stuurman, N.; Waller, L. Pycro-manager: open-source software for integrated microscopy hardware control and image processing. arXiv e-prints 2020, arXiv:2006.11330. 